\title{
Mathematical Models of Microwave and Photonic Devices Engaging Strong Nonlinearities Using Decomposition on Nonlinear Autonomous Blocks
}

\author{
G. S. MAKEEVA, O. A. GOLOVANOV \\ Department of Radioengineering \\ Penza State University, \\ 40, Krasnaya Street, Penza, 440026 \\ RUSSIA
}

\begin{abstract}
Mathematical modeling technique based on solving the nonlinear Maxwell's equations (Eqs.) rigorously using the decomposition approach on nonlinear autonomous blocks partially filled by the nonlinear media with a "strong" nonlinearity (NABs) and reliable engineering method for numerical computation of microwave and photonic nonlinear 3D devices engaging strong nonlinearities, applicable in $\mathrm{CAD}$, were developed. To determine the NAB descriptors the iterative computational process for solving the nonlinear 3D diffraction boundary problems with the non-asymptotic radiation boundary conditions on the NAB bounds was performed using the projection method. The iteration method of recomposition of NABs is developed using the linearization of its descriptors. Using the computational algorithm for solving nonlinear diffraction boundary problems performed as NABs and improved computation algorithm of determination of bifurcation points the nonlinearity thresholds in the magnetic nanoarrays at microwaves were numerically simulated.
\end{abstract}

Key-Words: - decomposition, engaging strong nonlinearities, nonlinear autonomous blocks, iterative computational process.

Received: September 2, 2020. Revised: February 23, 2021. Accepted: March 8, 2021. Published: March 17, 2021.

\section{Introduction}

There exists an increasing demand to develop new microwave 3D devices containing distributed insertions of nonlinear gyromagnetic and semiconductor media having a strong nonlinearity for high power applications and nonlinear analogue signal processing operating in radar detection, communication, and instrumentation.

Investigation of strong nonlinear effects: frequency multiplication, self-excited oscillations, parametric amplification and excitation, solitons, instability of electromagnetic (EM), magnetostatic and spin waves in 3D systems of arbitrary shaped magnetic nanoelements is important to design frequency multipliers, limiters, noise rejectors, frequency selective systems for microwave and magnetophotonic applications, nanosensor arrays, and Tbyte information storage [1].
A plethora of strong nonlinear effects in graphene in the teraherz (THz), infrared (IR) and optical frequency ranges, was successfully demonstrated, including saturable absorption and nonlinear refraction, higher harmonic generation and wavemixing processes, optical limiting, frequency mixing and frequency multiplication attracting increasing interest due to the possible applications for optical switching, novel optical sources, and harmonic frequency conversion, in ultrafast lasers and optical sensors [2]. These photonic devices are presently in the process of early development.

The development and manufacturing of microwave or photonic nonlinear 3D devices engaging strong nonlinearities depends on the development of computer aided design (CAD) tools, based on accuracy and the adequacy of mathematical models by solving Maxwell's equations (Eqs.) rigorously. Research and prediction 
of strong nonlinear physical phenomena and effects on these devices requires to develop new approaches for mathematical modeling.

The accurate EM modeling of nonlinear microwave and photonic devices by solving the nonlinear Maxwell's Eqs. rigorously is possible only using the decomposition approach [3], because of the complicated shape and the various size would cause computing problems.

Now the decomposition approach, consisting in division (or decomposition) of 3D devices into independently analyzed small volumes or autonomous blocks (Abs) with following recomposition of descriptors of $\mathrm{ABs}$, is used to solve linear problems of electrodynamics [3-6]. Descriptors of known ABs filled by linear homogeneous media (multimode ABs [4], minimal ABs [5], ABs with Floquet channels [6]) ABs in the form of multichannel multimode scattering (or conductivity, resistance) matrices have been calculated by solving the Maxwell's Eqs. complemented by simplified equations of motion in the material medium of the $\mathrm{AB}$ filling. However, these $\mathrm{AB}$ descriptors (S-, Y-, Z- matrices) can be used only in a case of $A B s$ filled by media with a weak degree of nonlinearity.

In general, commercially available FEM and FTDT software packages are used to design microwave and photonic devices. These packages are adequate of the technology level of the present day, but do not include the deep physical processes (including strong nonlinearities) upon which the nonlinear microwave and photonic devices of the near future will be based. Some relevant studies can be found in [7-9].

In our previous works linear or nonlinear interactions of EM waves with magnetic [10-13] and semiconductor [14] insertions in waveguides at microwaves and with graphene nanostructures $[15,16]$ at $\mathrm{THz}$ frequencies were numerically simulated by solving the Maxwell Eqs. complemented by simplified Eqs. of motion in the $\mathrm{AB}$ filling medium.

The goal of this work is to develop mathematical modeling technique based on solving of nonlinear boundary problems and reliable engineering method for numerical computation of microwave and photonic nonline Dr. Galina Makeeva,ar 3D devices engaging strong nonlinearities, applicable in CAD. In this paper a decomposition approach using ABs partially filled by nonlinear media with a "strong" nonlinearity (NABs) for modeling of nonlinear 3D devices was developed, based on accuracy and the adequacy of mathematical models by solving the nonlinear 3D diffraction boundary problems. The descriptors of NABs, containing "strong" nonlinear medium insertion, are determined as system of nonlinear Eqs., connecting magnitudes of incident and reflected modes on input cross-sections channels. At first the recomposition of NABs in accordance with the decomposition scheme of 3D devices is performed using the iterative computational process.

\section{Reduction the Nonstationary Nonlinear Maxwell's Equations to the System of Stationary Nonlinear Equations at Combination Frequencies}

The rigorous statement of the boundary problems for the nonlinear 3D devices filled by the nonlinear dielectric, semiconductor, conducting or magnetic media consists in the following. It is necessary to solve the nonlinear Maxwell's Eqs.

$$
\begin{gathered}
\operatorname{rot} \vec{H}(t)=\frac{\partial \vec{D}(\vec{E}(t))}{\partial t}+\vec{J}(\vec{E}(t)), \\
\operatorname{rot} \vec{E}(t)=-\frac{\partial \vec{B}(\vec{H}(t))}{\partial t},
\end{gathered}
$$

where $\vec{E}(t)=\vec{E}(x, y, z, t), \vec{H}(t)=\vec{H}(x, y, z, t)$ are the electric and magnetic field intensity vectors, $\vec{D}(\vec{E}(t))$ is the electric-flux density vector depending on the vector $\vec{E} ; \vec{B}(\vec{H}(t))$ is the magnetic induction vector depending on the vector $\vec{H} ; \vec{J}(\vec{E}(t))$ is the electric current density vector depending on the vector $\vec{E}$.

Considering an isotropic nonlinear media the dependences $\vec{D}(\vec{E}(t)), \vec{B}(\vec{H}(t)), \vec{J}(\vec{E}(t))$ may be determined using the scalar nonlinear functions: 


$$
\begin{aligned}
& \vec{D}(t)=\varepsilon_{0} \varepsilon(|\vec{E}(t)|) \vec{E}(t), \\
& \vec{B}(t)=\mu_{0} \mu(|\vec{H}(t)|), \\
& \vec{J}(t)=\sigma(|\vec{E}(t)|) \vec{E}(t),
\end{aligned}
$$

where $|\vec{E}(t)|,|\vec{H}(t)|$ are the moduli of the electric and magnetic field intensity vectors, correspondently; $\quad \varepsilon(|\vec{E}(t)|), \mu(|\vec{H}(t)|) \quad$ are the relative dielectric constant and magnetic permeability, as nonlinear functions of the modulus $|\vec{E}(t)|$ or $|\vec{H}(t)|, \quad \sigma(|\vec{E}(t)|) \quad$ is the nonlinear conductivity as the nonlinear function of the moduli $|\vec{E}(t)| ; \varepsilon_{0}, \mu_{0}$ are the electric or magnet constants.

Staying within the limits of the phenomenological approach, the nonlinear dependences $\vec{D}(\vec{E}(t)), \vec{B}(\vec{H}(t)), \vec{J}(\vec{E}(t))$ may be represented approximately by using the uniform approximation by the high order polynomials: $\varepsilon(|\vec{E}(t)|)=\varepsilon_{1}+\varepsilon_{2}|\vec{E}(t)|+\varepsilon_{3}|\vec{E}(t)|^{2}+\ldots+\varepsilon_{n}|\vec{E}(t)|^{n-1}$, $\mu(|\vec{H}(t)|)=\mu_{1}+\mu_{2}|\vec{H}(t)|+\mu_{3}|\vec{H}(t)|^{2}+\ldots+\mu_{n}|\vec{H}(t)|^{n-1}$, $\sigma(|\vec{E}(t)|)=\sigma_{1}+\sigma_{2}|\vec{E}(t)|+\sigma_{3}|\vec{E}(t)|^{2}+\ldots+\sigma_{n}|\vec{E}(t)|^{n-1}$ where the approximation coefficients are defined from the experiments.

Let's represent $\vec{E}(t), \vec{H}(t),|\vec{E}(t)|,|\vec{H}(t)|$ in the form of series of terms of the combination frequencies:

$$
\begin{aligned}
& \vec{E}(t)=\sum_{m=-\infty}^{\infty} \vec{E}\left(\omega_{m}\right) \exp \left(i \omega_{m} t\right), \vec{H}(t)=\sum_{m=-\infty}^{\infty} \vec{H}\left(\omega_{m}\right) \exp \left(i \omega_{m} t\right), \\
& |\vec{E}(t)|=\sum_{m=-\infty}^{\infty} E\left(\omega_{m}\right) \exp \left(i \omega_{m} t\right),|\vec{H}(t)|=\sum_{m=-\infty}^{\infty} H\left(\omega_{m}\right) \exp \left(i \omega_{m} t\right)
\end{aligned}
$$

where an index $\mathrm{m}$ is determined at ensemble of indices $\left\{m_{1}, m_{2}, \ldots, m_{r}\right\}, \mathrm{r}-\mathrm{a}$ number of harmonic transmitter sources.

Inserting (2), (3) into (1) and taking into account (4), we obtain the system of nonlinear stationary Maxwell's Eqs. at the combination frequencies:

$$
\operatorname{rot} \vec{H}\left(\omega_{m}\right)=i \omega_{m} \varepsilon_{0} \varepsilon_{1}\left(\omega_{m}\right) \vec{E}\left(\omega_{m}\right)+\vec{J}\left(\omega_{m}\right),
$$

$$
\operatorname{rot} \vec{E}\left(\omega_{m}\right)=-i \omega_{m} \mu_{0} \mu_{1}\left(\omega_{m}\right) \vec{H}\left(\omega_{m}\right)+\vec{Z}\left(\omega_{m}\right)
$$

where

$\vec{J}\left(\omega_{m}\right)=i \omega_{m} \varepsilon_{0}\left(\sum_{k=-\infty}^{\infty} \sum_{n=-\infty}^{\infty} \dot{\varepsilon}_{2}\left(\omega_{m}\right) \vec{E}\left(\omega_{k}\right) E\left(\omega_{n}\right) \gamma_{k n}+\right.$

$+\sum_{k=-\infty}^{\infty} \sum_{n=-\infty}^{\infty} \sum_{j=-\infty}^{\infty} \dot{\varepsilon}_{3}\left(\omega_{m}\right) \vec{E}\left(\omega_{k}\right) E\left(\omega_{n}\right) E\left(\omega_{j}\right) \gamma_{k n j}+$

$\left.+\sum_{k=-\infty}^{\infty} \sum_{n=-\infty}^{\infty} \sum_{j=-\infty}^{\infty} \ldots \sum_{l=-\infty}^{\infty} \dot{\varepsilon}_{n}\left(\omega_{m}\right) \vec{E}\left(\omega_{k}\right) E\left(\omega_{n}\right) E\left(\omega_{j}\right) \ldots E\left(\omega_{l}\right) \gamma_{k n j . . l}\right) ;$

$\vec{Z}\left(\omega_{m}\right)=i \omega_{m} \mu_{0}\left(\sum_{k=-\infty}^{\infty} \sum_{n=-\infty}^{\infty} \mu_{2}\left(\omega_{m}\right) \vec{H}\left(\omega_{k}\right) H\left(\omega_{n}\right) \gamma_{k n}+\right.$

$+\sum_{k=-\infty}^{\infty} \sum_{n=-\infty}^{\infty} \sum_{j=-\infty}^{\infty} \mu_{3}\left(\omega_{m}\right) \vec{H}\left(\omega_{k}\right) H\left(\omega_{n}\right) H\left(\omega_{j}\right) \gamma_{k n j}+$

$\left.\left.+\sum_{k=-\infty n=-\infty}^{\infty} \sum_{j=-\infty}^{\infty} \sum_{l=-\infty}^{\infty} \sum_{n}^{\infty} \mu_{m}\right) \vec{H}\left(\omega_{k}\right) H\left(\omega_{n}\right) H\left(\omega_{j}\right) \ldots H\left(\omega_{l}\right) \gamma_{k n j . . . l}\right) ;$

$\dot{\varepsilon}_{k}\left(\omega_{m}\right)=\varepsilon_{k}\left(\omega_{m}\right)-i \frac{\sigma_{k}\left(\omega_{m}\right)}{\varepsilon_{0} \omega_{m}}, k=1,2, \ldots, n ;$

$\gamma_{k n j \ldots l}=\left\{\begin{array}{cc}0, \text { if } & \omega_{k}+\omega_{n}+\omega_{j}+\ldots+\omega_{l} \neq \omega_{m}, \\ 1, \text { if } & \omega_{k}+\omega_{n}+\omega_{j}+\ldots+\omega_{l}=\omega_{m} .\end{array}\right.$

\section{Units Descriptor of Nonlinear Autonomous Block}

According to the decomposition approach, the region of $3 \mathrm{D}$ devices, where the EM problem is to be solved, is split by imaginary boundaries into subregions, which are referred to as linear or nonlinear $\mathrm{ABs}$ and regarded as "waveguide transformers" [3].

Consider nonlinear $\mathrm{ABs}$ in the form of rectangular parallelepiped partially filled by the nonlinear medium with a "strong" nonlinearity, having as the input sections the virtual multimode channel (NABs) (Fig. 1) as the "waveguide transformer" [3].

The dependence of the magnitudes $c_{k(\alpha)}^{-}\left(\omega_{m}\right)$ of reflected waves on the magnitudes $c_{n(\beta)}^{+}\left(\omega_{l}\right)$ of incident waves on the virtual channels of NABs is determined in the form of the system of nonlinear Eqs:

$$
\begin{aligned}
& c_{k(\alpha)}^{-}\left(\omega_{m}\right)=F_{k(\alpha)}\left(\omega_{m} ; c^{+}\right), \\
& \alpha=1,2, \ldots, 6, k=1,2, \ldots, N_{\alpha}, m= \pm 1,2, \ldots, \pm M,
\end{aligned}
$$


where $N_{\alpha}$ is the number of accounted eigenwaves in $\alpha$-th virtual channel, $M$ is the number of accounted combination frequencies, $c^{+}$is the argument determined at ensemble $\left\{c_{1(1)}^{+}\left(\omega_{1}\right), c_{1(2)}^{+}\left(\omega_{1}\right) \ldots, c_{n(\beta)}^{+}\left(\omega_{l}\right) N_{\beta}\right\}$.

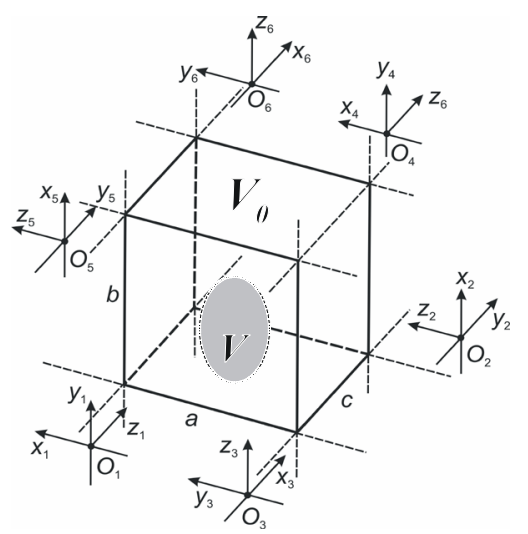

Fig.1. Nonlinear autonomous block with virtual channels. partially filled by the nonlinear medium (NAB): $V_{0}$ is the region inside the $\mathrm{AB} ; V$ is the region, filled by nonlinear medium with a "strong" nonlinearity; $V-V_{0}$ is the region, filled by a linear medium with relative permittivity $\varepsilon_{\mathrm{r}}$ and relative magnetic permeability $\mu_{\mathrm{r}}$

The nonlinear function $F_{k(\alpha)}\left(\omega_{m} ; c^{+}\right)$describing the dependence of magnitudes $c_{k(\alpha)}^{-}\left(\omega_{m}\right)$ on magnitudes $c_{n(\beta)}^{+}\left(\omega_{l}\right)$ on the input cross-sections of NABs may be used alternatively as the NAB descriptor.

The computational algorithm to determine the NAB descriptors was created using the numerical method of simple iteration [17]. For every step of iteration it is necessary to solve the nonlinear Maxwell's Eqs (1) with the non-asymptotic radiation boundary conditions [18] on NAB bounds:

$$
\begin{aligned}
& \int_{S_{\alpha}}\left(\vec{E}\left(\omega_{m}\right) \times \vec{h}_{k(\alpha)}\left(\omega_{m}\right)+\vec{e}_{k(\alpha)}\left(\omega_{m}\right) \times \vec{H}\left(\omega_{m}\right)\right) d \vec{s}_{\alpha}= \\
& =2 c_{k(\alpha)}^{+}\left(\omega_{m}\right) R_{k(\alpha)}\left(\omega_{m}\right)
\end{aligned}
$$

where $\vec{e}_{k(\alpha)}\left(\omega_{m}\right), \vec{h}_{k(\alpha)}\left(\omega_{m}\right)$ are the transverse components of electric and magnetic fields of eigenwaves in virtual channels, $R_{k}\left(\omega_{m}\right)$ is the normalization coefficient.
At first step of iteration the computational algorithm for calculating the scattering matrix $\mathbf{S}$ of NAB (Fig.1) was developed by solving the 3D diffraction boundary problem using the Galerkin projection method [17]. We use the eigenwaves of the rectangular resonator (Fig. 1) as the basis functions and determine the eigenfrequencies and the eigenwaves of the resonator by solving the homogenous Maxwell's Eqs with periodic boundary conditions on the NAB bounds (Fig.1). We find the solution of the 3D diffraction problem for the stationary Maxwell's Eqs. (5) with the boundary conditions (7) in the form of Fourier's series, using the eigenwaves of resonator inside the NAB region (Fig. 1), or eigenwaves of channels on the NAB cross-sections $\mathrm{S}_{\beta}$ (Fig. 1). The tangential EM field on each NAB cross-section $S_{\beta}$ is represented as a superposition of eigenwaves of channels [6]. When the magnitudes $c_{n(\beta)}^{+}\left(\omega_{l}\right)$ are known, the unknown magnitudes $c_{k(\alpha)}^{-}\left(\omega_{m}\right)$ can be found. Substituting the Fourier's series into the Eqs (5), which are represented in projection integral form [19], we obtain a system of nonlinear algebraic Eqs. These algebraic Eqs. are solved by using the Newton's method [17].

Using the iterative method we determine the NAB descriptor at every step of iteration by using the descriptors of linearized $\mathrm{AB}$ in the form the multimode S-matrix. The magnitudes $c_{n(\beta)}^{+}\left(\omega_{m}\right)$ and the currents $\vec{J}\left(\omega_{m}\right), \vec{Z}\left(\omega_{m}\right)$, localized in the volume of parallelepipeds (Fig. 1), are known for every iteration; the magnitudes $c_{k(\alpha)}^{-}\left(\omega_{m}\right)$ are unknown.

The solution of the nonlinear Maxwell's Eqs. (5) with the boundary conditions (7) is represented in the matrix form:

$$
\mathrm{C}^{-}=\mathrm{C}_{\Sigma}^{-}+\mathrm{SC}^{+}
$$

where $S$ is the multimode scattering matrix of linearized $\mathrm{AB}$ (the analytical expressions of $\mathrm{S}$ parameters of linear $\mathrm{AB}$ with virtual Floquet channels were obtained in [6]), $C_{\Sigma}^{-}$is a radiation vector determined by using the exciting currents 
$\vec{J}\left(\omega_{m}\right), \vec{Z}\left(\omega_{m}\right) ; \quad C^{+}, C^{-}$are the vectors with components

$$
c_{n(\beta)}^{+}\left(\omega_{m}\right), \quad c_{k(\alpha)}^{-}\left(\omega_{m}\right),
$$

correspondingly.

Solving the EM problem of excitation of waves in the rectangular cavity (Fig.1) by using the method, described in details in [20], we obtain the following system of linear algebraic Eqs. (SLAE) for determining of components of the radiation vector $C_{\Sigma}^{-}$:

$$
\left(\mathrm{I}+\mathrm{Y}^{\mathrm{T}}\right) \mathrm{C}_{\Sigma}^{-}=\mathbf{f}
$$

where $\mathrm{I}$ is the unitary matrix, $\mathrm{Y}^{T}$ is the transposed conductance matrix $\mathbf{Y}$ of $\mathrm{AB}$, filled with linear media [3], $f$ is the vector with the components determined as

$$
\begin{aligned}
& f_{k(\alpha)}\left(\omega_{m}\right)=-\int_{V}\left(\vec{J}\left(\omega_{m}\right) \cdot \vec{E}_{k(\alpha)}\left(\omega_{m}\right)+\right. \\
& \left.+\vec{Z}\left(\omega_{m}\right) \cdot \vec{H}_{k(\alpha)}\left(\omega_{m}\right)\right) d V
\end{aligned}
$$

As a result the magnitudes $c_{k(\alpha)}^{-}\left(\omega_{m}\right)$ are determined for each step of iteration.

For the transition to the next iteration it is necessary to redefine the currents $\vec{J}\left(\omega_{m}\right), \vec{Z}\left(\omega_{m}\right)$ by using the projection method [17] to determine the EM fields $\vec{E}\left(\omega_{m}\right), \vec{H}\left(\omega_{m}\right)$ in the rectangular cavity (Fig.1) having bounds as magnetic or electric walls.

\section{Recomposition of Nonlinear Autonomous Blocks}

In order to realize the recomposition of NABs (or nonlinear $\mathrm{ABs}$ with linear $\mathrm{ABs}$ ) for $3 \mathrm{D}$ devices it is necessary to solve the system of nonlinear Eqs (6). The algorithmization of this complex computational procedure is difficult. That's why the iterative method of the recomposition of NABs, based on the linearization of its descriptors, is proposed. During every iteration we replace NABs by linearized $A B s$ with descriptors as multimode S-matrices. After that the recomposition of NABs (or NABs and $\mathrm{ABs}$ ) in accordance with the decomposition scheme [3] of $3 \mathrm{D}$ devices is performed.

Let's represent the nonlinear functions
$F_{k(\alpha)}\left(\omega_{m} ; c^{+}\right)(6)$ with their generalized Taylor's series:

$$
\begin{aligned}
& c_{k(\alpha)}^{-}\left(\omega_{m}\right)=F_{k(\alpha)}\left(\omega_{m} ; c_{0}^{+}\right)= \\
& =\sum_{l=1}^{M} \sum_{\beta=1}^{L} \sum_{n=1}^{N_{\beta}} \frac{\partial F_{k(\alpha)}\left(\omega_{m} ; c_{0}^{+}\right)}{\partial c_{n(\beta)}^{+}\left(\omega_{l}\right)}\left(c_{n(\beta)}^{+}\left(\omega_{l}\right)-c_{n(\beta)}^{+0}\left(\omega_{l}\right)\right)+\ldots,
\end{aligned}
$$

where $M$ is the number of the accounted combination frequencies, $L$ is the number of virtual channels, $N_{\beta}$ is the number of the accounted eigenwaves in $\beta$-th virtual channel .

Taking into account the partial derivatives up to the first order in (10), as the first approximation, let's reduce the system of nonlinear Eqs to SLAE. It results the linearized dependence of magnitudes of reflected modes $c_{k(\alpha)}^{-}\left(\omega_{m}\right)$ on magnitudes of incident mode $c_{n(\beta)}^{+}\left(\omega_{l}\right)$ and it may be represented in the form:

$$
\mathbf{C}^{-}=\mathbf{C}_{\Sigma}^{-}+\mathbf{S}_{\mathbf{H}} \mathbf{C}^{+}
$$

where $C_{\Sigma}^{-}=C_{0}^{-}-S_{H} C_{0}^{+}$.

The components of vectors $C_{0}^{+}, C_{0}^{-}, C^{+}, C^{-}$ are $\left\{c_{n(\beta)}^{+0}\left(\omega_{l}\right)\right\}, \quad\left\{F_{k(\alpha)}\left(\omega_{m} ; c_{o}^{+}\right\}, \quad\left\{c_{n(\beta)}^{+}\left(\omega_{l}\right)\right\}\right.$, $\left\{c_{k(\alpha)}^{-}\left(\omega_{m}\right)\right\}$, correspondingly. The scattering matrix $S_{H}$ is arranged on the combination frequencies, the input channels, the types of eigenwaves. The Sparameters of matrix $S_{H}$ are determined in the following way:

$$
S_{k n}^{\alpha \beta}\left(\omega_{m}, \omega_{l}\right)=\frac{\partial F_{k(\alpha)}\left(\omega_{m} ; c_{0}^{+}\right)}{\partial c_{n(\beta)}^{+}\left(\omega_{l}\right)}
$$

The components of the vector $C_{\Sigma}^{-}$are $\left\{c_{\Sigma k(\alpha)}^{-}\left(\omega_{m}\right)\right\}$, the radiation vector $C_{\Sigma}^{-}$is arranged in the same way as the vector $C^{-}$.

The linearized $\mathrm{ABs}$ are included in the decomposition scheme by using the additional fictive ABs with descriptors as the scattering matrix [3]. Using the fictive $A B s$ the recognizing access for components of the vectors $C_{\Sigma}^{-}$and $C^{+}, C^{-}$is 
realized. It is necessary to organize and control the computational process.

The iterative computational process of recomposition of NABs consists in the following. Specifying the initial approximation for the value $c_{0}^{+}$we determine S-parameters of matrices $S_{H}$ and the components of vectors $C_{\Sigma}^{-}$for all NABs included in the decomposition scheme of 3D devices. By connecting the descriptors of linearized ABs as the scattering matrix $S_{H}$ we obtain the resulting sum S-matrix of nonlinear 3D device for the first iteration. If the magnitudes of incident waves in input channels of 3D device are known we determine the magnitudes of reflected modes and the components of the vector $C^{+}, C^{-}$in virtual channels.

After every iteration we verify the results of computing in the following way. If the values of components of vectors $C^{+}, C^{-}$are satisfied with a prescribed accuracy to the nonlinear Eqs. (6), the iterative computational process is finished. If not, at the next iteration the S-parameters of matrices $S_{H}$ and the components of the vector $C_{\Sigma}^{-}$are determined assigning the initial approximation $C_{0}^{+}=C^{+}$from (11), (12) and the process of recomposition of linearized $\mathrm{ABs}$ is repeated.

\section{Numerical Simulation of Nonlinearity Thresholds in Magnetic Nanoarrays}

Let us consider, as an example, the problem of nonlinear diffraction of EM waves by magnetic nanoarrays of ferromagnetic nanoparticles. To solve the nonlinear diffraction boundary problem a computational algorithm was performed based on the decomposition approach by NABs developed by us.

For the calculations we consider the model of the elementary cell of the 2D periodic magnetic nanoarray, where each cell contains one ferromagnetic spherical nanoparticle (Fig.1) (or any other shape of a nanoparticle) as a NAB. The cell is described by its NAB descriptor, taking into account electrodynamic boundary conditions, the geometry of the array, and the shape of magnetic nanoparticle.

To determine the NAB descriptors the computational algorithm was developed by solving the nonlinear 3D diffraction boundary problem for the Maxwell's equations using electrodynamic boundary conditions, complemented by the LandauLifshitz equation of motion of the magnetization vector in a ferromagnet, including the exchange term [21].

For the analysis of strong nonlinear phenomena (in particular, the parametric instability) in magnetic nanoarrays the numerical method, using the bifurcation points of the nonlinear Maxwell's operator, developed by us [22, 23], is applied.

The results of computing the instability regions for the parametric excitation of magnetostatic and spin waves in the nonlinear magnetic nanoarrays by the incident pumping wave depending on the bifurcation parameters, i.e. the magnitude of the pumping wave $\mathrm{C}^{+1(1)}\left(\omega_{\mathrm{H}}\right)$ and the normalized frequency (frequency of the signal wave $\omega_{0}$ with respect to the pumping frequency $\omega_{\mathrm{H}}$ ), for various separations $h$ of ferromagnetic nanoparticles are shown in Fig. 2.

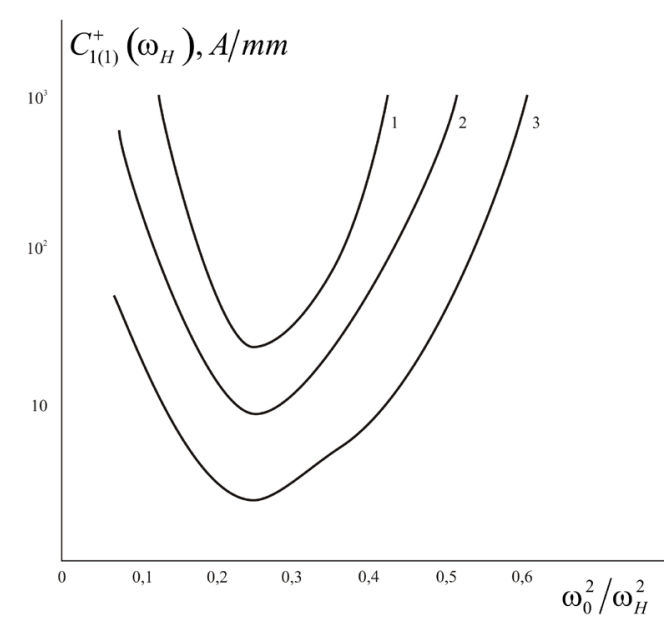

Fig. 2. Nonlinearity thresholds in the nonlinear magnetic nanoarrays of ferromagnetic nanoparticles $(\mathrm{r}=250 \mathrm{~nm})$ for different separation $\mathrm{h}$ : curves 1$\mathrm{h}=3000 \mathrm{~nm} ; 2-750 \mathrm{~nm} ; 2-650 \mathrm{~nm} ; \omega_{0}=2 \pi \mathrm{f}_{0}-$ frequency of the signal wave; $\mathrm{f}_{0}=9.330 \mathrm{GHz} ; \omega_{\mathrm{H}}-$ frequency of the pumping wave; $\mathrm{C}^{+1(1)}\left(\omega_{\mathrm{H}}\right)$ magnitude of the incident pumping wave; bias field; $H_{0}=\omega / \gamma, M_{0}=0.026 \mathrm{~T}$

The threshold magnitudes $\mathrm{C}^{+1(1)}\left(\omega_{\mathrm{H}}\right)$ of pumping EM wave (the curves in Fig. 2), where the strong nonlinear processes occur, are determined by computing the bifurcation points of the nonlinear Maxwell's operator. 
According to the Lyapunov stability theory the curves (shown in Fig. 2) divide the instability regime of the parametric excitation of magnetostatic and spin waves (above the curves) from the stable regime of parametric amplification (below the curves) [22].

The results of numerical simulation show that the nonlinearity thresholds are reduced at regime of parametric instability, with decreasing separation into the nm range the instabilities occur at lower inputs, demonstrating the power sensitivity of magnetic nanoarrays.

\section{Conclusion}

We develop the electrodynamical analysis methodology, differing from the known ones, that it is based on rigorous solving of Maxwell's equations complemented by the Eqs. of motion in the nonlinear material medium of the $\mathrm{AB}$ filling without any simplification of Eqs. and boundary conditions.

This methodology uses the decompositional approach, that's why it is possible to model nonlinear 3D devices having complex geometry, to make a quick transition from one boundary value problem to other ones, to alleviate numerical problems, connected with the instability of computing processes. That's why the developed computational algorithms for calculation of Smatrices of an NAB can be used for the electrodynamic analysis of microwave- and photonic nonlinear 3D devices containing inclusions of the nonlinear media with a "strong" nonlinearity of arbitrary shapes.

The developed decomposition approach by using NABs can be applied for numerical simulation of time-stable and spatially nonuniform processes (with large amplitudes) in nonlinear microwave and photonic 3D devices taking account costrained geometries. Modeling unstable nonlinear phenomena necessitates the NAB-based algorithm and the improved computations algorithm of determination of bifurcation points of the nonlinear Maxwell operator.

The developed adequate models based on the solution of the nonlinear Maxwell equations (where the coefficients of strong nonlinearities of material media are defined from the experiments) without any simplifications of the equations and the boundary conditions make it possible to abandon the empirical approach to design the nonlinear microwave and photonic 3D devices.

The computational algorithms for solving diffraction boundary problems using numerical methods of NABs can be included in software package, intended to be developed in the future for mathematical simulation and $\mathrm{CAD}$ of perspective microwave and photonic $3 \mathrm{D}$ devices using strong nonlinear phenomena.

\section{References:}

[1] S A Nikitov, D.V.Kalyabin, I.V.Lisenkov, A.N. Slavin et al. "Magnonics: a new research area in spintronics and spin wave electronics" UFN, Vol.185: No.10 (2015), pp. 1099-1128.

[2] D. Turchinovich, L. Caspani, A. TaukePedretti, F. Leo, and B. Yang,"Terahertz Nonlinear Optics of Graphene, Probably the Most Nonlinear Material We Know," $A d v$. Optical Mater. Vol.8, 2020, pp.1900771.

[3] V. Nikol'skii and T.I. Nikol'skaya. Decomposition Approach in Electrodynamics Problems. Nauka, Moscow, 1983 [in Russian].

[4] V. V. Nikol'skii and O. A. Golovanov, Autonomous multimode blocks and its application for the research of strip-lines. Radiotekh. Elektron. Vol. 24, 1979, pp. 1070.

[5] V. V. Nikol'skii and T. I. Lavrova, The method of minimum autonomous blocks and its application to waveguide diffraction problems. Radiotekh. Elektron. Vol. 23, 1978, pp. 241.

[6] O. A. Golovanov, "Solution of Applied Problems of Electromagnetics Based on the Use of Autonomous Blocks with Virtual Floquet Channels", J. Commun. Techn. Electron, Vol. 51, 2006, pp. 1423-1430.

[7] Jin, C. Argyropoulos. Nonlinear graphene metasurfaces with advanced electromagnetic functionalities. Proc. SPIE. 10722, Plasmonics: Design, Materials, Fabrication, Characterization, and Applications XVI, 107221R, 2018; doi:10.1117/12.2319878.

[8] J. C. Deinert, D. A. Iranzo, R. Pérez et al. Grating-graphene metamaterial as a platform for terahertz nonlinear photonics. ACS Nano,., Vol. 15, 1, 2021 1145-1154. https://doi.org/10.1021/acsnano.0c08106

[9] D. Cox and F.J. García De Abajo, "Nonlinear Graphene Nanoplasmonics," Accounts of Chemical Research, Vol. 52, No.9, 2019, pp. 2536-2547. 
[10] G.S.Makeeva， O.A.Golovanov， M.PardaviHorvath.An Efficient Nonlinear Frequency Multiplication Mechanism in Ferrite Loaded Waveguide Structures, IEEE Transaction on Magnetics, Vol. 41. No.10, 2005, pp.35593561.

[11] M.Pardavi-Horvath. G.S.Makeeva, O.A.Golovanov. Nonlinear Phenomena in Magnetic Nanoparticle Systems at Microwave Frequencies, IEEE Transaction on Magnetics. . 2008. Vol.44. No.11, 2008. pp. 3067-3070.

[12] M. Pardavi-Horvath, G. S. Makeeva, O. A. Golovanov. Electrodynamic Model for Low Loss Microwave Nanocomposite Magnets Using Autonomous Blocks with Floquet Channels, Journal of Applied Physics, Vol. 105, Issue 7, 2009, pp. 07C104-07C104-2.

[13] Makeeva G. S. Pardavi-Horvath M. Golovanov O.A. Tuning the Scattering Parameters of Magnetic Nanowire Arrays Near the Antiresonance at Photonic Frequencies. IEEE Transaction on Magnetics, Vol. 45, No. 10, 2009. pp. 4074-4076.

[14] G. S. Makeeva, O. A. Golovanov, PardaviHorvath. Numerical Simulation of Nonlinear and Parametric Oscillations in a Semiconductor Resonator Structure. J. Electromagnetic Waves and Applications (JEMWA), Vol. 20, No.11, 2006, pp. 1516-1520.

[15] G. S. Makeeva and O. A. Golovanov. Numerical Modeling of Reconfigurable $\mathrm{THz}$ Devices Based on Graphene Nanostructures Using Autonomous Blocks with Floquet Channels. Proceedings of the 45th European Microwave Conference (EuMC) European Microwave Week 2015 (EuMW2015. Paris, France. 7-10 September 2015. pp. 1061 1064.

[16] O. A. Golovanov, G. S. Makeeva, and A. B. Rinkevich. Interaction of Terahertz Electromagnetic Waves with Periodic Gratings of Graphene Micro- and Nanoribbons. Technical Physics. 2016, Vol. 61, No. 2, pp. 274-282.

[17] N. S. Bahvalov. Numerical methods. Moscow. Nauka.1975. (in Russian)

[18] O. A. Golovanov, "Numerical algorithm for solving the problems of diffraction for microwave waveguides with nonlinear media", Radiotekhnika i elektronika, [J. Comm. Tech. and Electronics], Vol. 35, 1990, pp.1853-1863.

[19] V. V. Nikolskii, Projection methods in electrodynamics, Collection of papers on applied electrodynamics, Visshaya Skola, Moscow, 1977 (in Russian)
[20] L. A. Vainstein Electromagnetic waves. Moscow. Radio i sviaz.1988. (in Russian)

[21] O. A. Golovanov and G. S. Makeeva. "The Method of Autonomous Blocks with Magnetic Nanoinclusions and Floquet Channels Applied for Simulation of Magnetic Nanostructures with Allowance for the Exchange and Boundary Conditions". J. Commun. Techn. Electron. (Radiotekhnika $i$ Elektronika), Vol.54, 2009 p. 1345.

[22] G. S. Makeeva, O. A. Golovanov, M. PardaviHorvath, "Numerical Analysis of Electromagnetic Wave Instability in Nonlinear Ferrite Structures Using Bifurcation Points of the Nonlinear Maxwell's Operator", IEEE Trans. Mag., Vol.. 42, No.10, 2006, pp. 35503552.

[23] G. S. Makeeva and O. A. Golovanov, "Numerical Investigation of Instabilities of Waves and Oscillations in Nonlinear Gyromagnetic Structures with the Use of Bifurcation Points of the Nonlinear Maxwell Operator", J. of Communic. Techn. Electron., vol. 52, 2007 pp. 96-103.

\section{Contribution of individual authors to the creation of a scientific article (ghostwriting policy)}

Galina S. Makeeva developed the methodology and mathematical modeling technique.

Oleg A. Golovanov has implemented the Algorithms and carried out the simulation.

John Smith, Donald Smith carried out the simulation

\section{Creative Commons Attribution License 4.0 (Attribution 4.0 International, CC BY 4.0)}

This article is published under the terms of the Creative Commons Attribution License 4.0

https://creativecommons.org/licenses/by/4.0/deed.en_US 